\title{
Study on MIMO-based spectrum sharing with stream allocation error
}

\author{
Samuli Tiiro ${ }^{\text {a) }}$, Kenta Umebayashi, and Yasuo Suzuki \\ Graduate School of Engineering, Tokyo University of Agriculture and Technology, \\ Naka-cho, Koganei-shi, Tokyo, 2-24-16, Japan \\ a) $50011834205 @$ st.tuat.ac.jp
}

\begin{abstract}
In order to achieve more efficient overall spectrum utilization, it is possible to employ cognitive beamforming for secondary spectrum access. An important issue in such spatial spectrum sharing techniques is protecting the primary user from interference which arises due to estimation errors when the secondary users try to locate the available transmit opportunities. In this paper, we investigate the issue of primary user protection when the secondary user allocates too many spatial streams for the secondary transmission. We provide analysis for the interference power statistics and take advantage of the moment matching method in order to obtain an expression for the interference power that is tractable for practical analysis. In addition, we propose a protection constraint which limits the resulting interference and protects the primary user in terms of interference outage probability. We also show the achievable transmit power for the secondary user which can satisfy the protection constraint.
\end{abstract}

Keywords: cognitive radio, interference, MIMO, spectrum sharing Classification: Terrestrial Wireless Communication/Broadcasting Technologies

\section{References}

[1] F. Gao, R. Zhang, Y.-C. Liang, and X. Wang, "Design of Learning-Based MIMO Cognitive Radio Systems," IEEE Trans. Veh. Technol., vol. 59, no. 4, pp. 1707-1720, May 2010.

[2] P. G. Moschopoulos, "The Distribution of the Sum of Independent Gamma Random Variables," Annals of the Institute of Statistical Mathematics (Part A), vol. 37, no. 3, pp. 541-544, 1985.

[3] R. W. Heath Jr., T. Wu, Y. H. Kwon, and A. C. K. Soong, "Multiuser MIMO in Distributed Antenna Systems With Out-of-Cell Interference," IEEE Trans. Signal Process., vol. 59, no. 10, pp. 4885-4899, Oct. 2011.

[4] S. Tiiro, K. Umebayashi, and Y. Suzuki, "Decision Fusion for Cooperative Source Number Estimation in Cognitive Radio Networks," IEICE Commun. Express, vol. 2, no. 11, pp. 484-489, Nov. 2013. 


\section{Introduction}

Cognitive radio (CR) techniques have attracted significant amount of interest in the recent years as a possible solution to the spectrum scarcity problem. Especially interesting are beamforming methods that can spatially interleave the secondary transmissions so that interference to the primary user (PU) can be avoided. Ideally, when the secondary user (SU) is equipped with multiple transmit antennas, it is possible to transmit concurrently with the PU while negating all interference to the PU.

In practical systems, there are two important issues that result in harmful interference. First is using imperfect channel state information (CSI) when designing the SU's beamforming matrix and the second one is the SU incorrectly allocating too many streams for secondary transmission. While the former is a major issue and it has been studied in the literature [1], the latter has not been investigated deeply.

In this paper, we consider the issue of avoiding interference in a CR network with incorrect allocation of secondary transmit streams. We carry out analysis of the interference power statistics so that it is possible to satisfy an interference outage based protection constraint by regulating the transmit power at the SU terminal. Furthermore, we evaluate the achievable SU transmit power under the proposed protection constraint.

\section{System model and background}

For the SU, we consider a transmitter-receiver pair where both terminals have $M_{\mathrm{SU}}$ antennas. We also have one PU terminal with $M_{\mathrm{PU}}$ antennas. The PU is assumed to alternate between transmit and receiving modes so that the SU can estimate the available transmit opportunities during the primary transmissions and correspondingly protect the $\mathrm{PU}$ from interference while it is in receiving mode. The channel between the secondary transmitter and the PU is denoted by $G \in \mathbb{G}^{M_{\mathrm{PU}} \times M_{\mathrm{SU}}}$ and the secondary transmission channel is denoted by $\boldsymbol{H} \in \mathbb{C}^{M_{\mathrm{SU}} \times M_{\mathrm{SU}}}$. The channels are considered to be Rayleigh fading channels with the channel elements distributed as $\mathcal{C N}(0,1)$.

Spatial secondary spectrum access can be realized by having the SU map its signals onto the null space of the interference channel. In practice, the SU has to estimate the null space in order to compute the corresponding beamforming matrix $\boldsymbol{A} \in \mathbb{C}^{M_{\mathrm{SU}} \times d}$. The dimension of the null space dictates the number of spatial streams that can support orthogonal secondary transmission. To maximize the secondary throughput, the goal is to use all of the available streams, the number of which equals to $d=M_{\mathrm{SU}}-M_{\mathrm{PU}}$.

When the PU parameters are not known, the SU has to estimate $d$ to determine the available spatial transmit resources. With estimated $d$, it is possible that an erroneous estimate will result in interference towards the PU. In the case $\hat{d}<d$, the SU will not use all available spatial dimensions. While this results in suboptimal capacity for the SU, it will not cause interference to the PU. However, if $\hat{d}>d$ the SU's transmit signals will overlap with PU's signal space and cause significant amount of interference. This is 
because when the available transmit space dimension is overestimated, additional vectors which are not orthogonal to $\boldsymbol{G}$ are chosen to be included in the estimated null space. As a result, the singular vectors forming the beamforming matrix $\boldsymbol{A}$ completely change.

In this paper, we study the resulting interference when $\hat{d}>d$. To protect the PU from interference, we define a constraint in terms of interference outage probability

$$
\operatorname{Prob}\left(I_{P} \leq \gamma\right) \geq 1-P_{\text {out }}
$$

where $I_{P}$ denotes the interference power, $\gamma$ is the interference power threshold, and $P_{\text {out }}$ is the desired outage probability. The goal is then to find the maximum transmit power the $\mathrm{SU}$ can use while satisfying the protection constraint.

\section{Interference with stream allocation error}

After the channel learning, the SU has obtained an estimate for the available transmit streams, $\hat{d}$, and corresponding beamforming matrix $\boldsymbol{A} \in \mathbb{C}^{M_{\mathrm{SU}} \times \hat{d}}$. Now, the interference power at the PU can be expressed as

$$
I_{P}=\mathcal{E}\left\{\left\|\boldsymbol{G} \boldsymbol{A} \boldsymbol{x}_{\mathrm{SU}}[n]\right\|_{2}^{2}\right\}=\mathcal{E}\left\{\operatorname{trace}\left(\boldsymbol{G} \boldsymbol{A} \boldsymbol{x}_{\mathrm{SU}}[n] \boldsymbol{x}_{\mathrm{SU}}^{H}[n] \boldsymbol{A}^{H} \boldsymbol{G}^{H}\right)\right\} .
$$

Here, slow fading scenario is assumed where the channel is considered constant during a single transmission frame. The transmitted secondary signals have $\mathcal{E}\left\{\left\|\boldsymbol{x}_{\mathrm{SU}}[n]\right\|_{2}^{2}\right\}=\boldsymbol{\Sigma}$, where $\boldsymbol{\Sigma}=\operatorname{diag}\left(\sigma_{1}^{2} \ldots \sigma_{\hat{d}}^{2}\right)$ and $\sigma_{i}^{2}$ denotes the transmit power of the $i$ th stream. The maximum transmit power constraint for the $\mathrm{SU}$ is $P_{\mathrm{t}}$ and therefore $\operatorname{trace}(\boldsymbol{\Sigma}) \leq P_{\mathrm{t}}$. Now, the interference can be rewritten as

$$
I_{P}=\operatorname{trace}\left(\boldsymbol{\Sigma} \boldsymbol{A}^{H} \boldsymbol{G}^{H} \boldsymbol{G} \boldsymbol{A}\right) .
$$

To satisfy the protection constraint (1), the distribution of $I_{P}$ is required. From (3) it can be seen that $\boldsymbol{G}^{H} \boldsymbol{G}$ is a Wishart matrix with distribution $\mathcal{C} \mathcal{W}_{M_{\mathrm{SU}}}\left(M_{\mathrm{PU}}, \boldsymbol{I}_{M_{\mathrm{SU}}}\right) . I_{P}$ can also be written as

$$
I_{P}=\sigma_{1}^{2} \boldsymbol{a}_{1}^{H} \boldsymbol{G}^{H} \boldsymbol{G} \boldsymbol{a}_{1}+\cdots+\sigma_{\hat{d}}^{2} \boldsymbol{a}_{\hat{d}}^{H} \boldsymbol{G}^{H} \boldsymbol{G} \boldsymbol{a}_{\hat{d}}
$$

where $\boldsymbol{a}_{i}$ is the $i$ th column vector of the beamforming matrix. From the property of Wishart matrices it follows that $\boldsymbol{a}_{i}^{H} \boldsymbol{G}^{H} \boldsymbol{G} \boldsymbol{a}_{i}$ follow distribution $\Gamma\left(M_{\mathrm{PU}}, \boldsymbol{a}_{i}^{H} \boldsymbol{a}_{i}=1\right)$ and due to the scaling property of the Gamma distribution $\sigma_{i}^{2} \boldsymbol{a}_{i}^{H} \boldsymbol{G}^{H} \boldsymbol{G a}_{i} \sim \Gamma\left(M_{\mathrm{PU}}, \sigma_{i}^{2}\right)$ follows.

While exact expressions are available for the distribution of a sum of Gamma distributed variables [2], they are not very useful for practical applications. Therefore, we approximate the distribution with another Gamma distribution by matching its moments to the sum of the corresponding moments of the coefficients [3]. Denote the r.v. used to approximate $I_{P}$ by $X \approx \sum_{i=1}^{\hat{d}} \sigma_{i}^{2} \boldsymbol{a}_{i}^{H} \boldsymbol{G}^{H} \boldsymbol{G a}_{i}$. Now the two first moments of $X$ are

$$
\mu_{X}=\sum_{i=1}^{\hat{d}} M_{\mathrm{PU}} \sigma_{i}^{2}=M_{\mathrm{PU}} \sum_{i=1}^{\hat{d}} \sigma_{i}^{2}=M_{\mathrm{PU}} P_{\mathrm{t}}
$$


and

$$
\sigma_{X}^{2}=\sum_{i=1}^{\hat{d}} M_{\mathrm{PU}}\left(\sigma_{i}^{2}\right)^{2}=M_{\mathrm{PU}} \sum_{i=1}^{\hat{d}}\left(\sigma_{i}^{2}\right)^{2}
$$

which follows from the two first moments of a r.v. having distribution $\Gamma(k, \theta)$ being $k \theta$ and $k \theta^{2}$, respectively. Furthermore, the distribution for the approximation becomes $\Gamma\left(k_{X}=\frac{\mu_{X}^{2}}{\sigma_{X}^{2}}, \theta_{X}=\frac{\sigma_{X}^{2}}{\mu_{X}}\right)$.

To determine the maximum transmit power $P_{\mathrm{t}}$ that satisfies the protection constraint, the cumulative distribution function for $X$ is needed

$$
F_{X}\left(x ; k_{X}, \theta_{X}\right)=\frac{1}{\Gamma\left(k_{X}\right)} \int_{0}^{\frac{x}{\theta_{X}}} t^{k_{X}-1} e^{-t} d t .
$$

To satisfy the protection constraint (1) for given interference threshold $\gamma$ we get

$$
\operatorname{Prob}(X \leq \gamma)=F_{X}(\gamma)=\frac{1}{\Gamma(k)} \int^{\frac{\gamma}{\theta_{X}}} t^{k_{X}-1} e^{-t} d t=1-P_{\text {out }},
$$

and $P_{\mathrm{t}}$ can be solved by finding a limit $x_{0}=\frac{\gamma}{\theta_{X}}$ for the integral which results in the integrand being equal to $1-P_{\text {out }}$.

Now, consider the parameters for $X$

$$
k_{X}=\frac{\mu_{X}^{2}}{\sigma_{X}^{2}}=\frac{\left(M_{\mathrm{PU}} P_{\mathrm{t}}\right)^{2}}{M_{\mathrm{PU}} \sum_{i=1}^{\hat{d}}\left(\sigma^{2}\right)^{2}}=\frac{M_{\mathrm{PU}} P_{\mathrm{t}}^{2}}{\sum_{i=1}^{\hat{d}}\left(\sigma_{i}^{2}\right)^{2}}
$$

and

$$
\theta_{X}=\frac{M_{\mathrm{PU}} \sum_{i=1}^{\hat{d}}\left(\sigma_{i}^{2}\right)^{2}}{M_{\mathrm{PU}} P_{\mathrm{t}}}=\frac{\sum_{i=1}^{\hat{d}}\left(\sigma_{i}^{2}\right)^{2}}{P_{\mathrm{t}}} .
$$

We notice two issues that complicate the problem of finding maximal $P_{\mathrm{t}}$. First, both parameters depend on $M_{\mathrm{PU}}$ for which only an incorrect estimate (in the case of stream allocation error) is available at the SU. In addition, the parameters also depend on the power allocations for the individual streams which in turn depend on $P_{\mathrm{t}}$. In practice, $M_{\mathrm{SU}}$ can be used as the worst case approximation of $M_{\mathrm{PU}}$. While this serves as a safeguard against the interference towards the PU, it will result in suboptimal SU capacity. However, we ignore this and evaluate the theoretically achievable maximum transmit power. For the second issue, we consider two different cases. The term $\sum_{i=1}^{\hat{d}}\left(\sigma_{i}^{2}\right)^{2}$ achieves its maximum $P_{\mathrm{t}}^{2}$ when all the transmit power is allocated for a single stream (SS). On the other hand, minimum $\frac{P_{\mathrm{t}}^{2}}{\hat{d}}$ is achieved when equal powers are allocated for each stream (UPA). Here, we evaluate the maximum transmit powers for both methods. The resulting parameters for the interference power distribution are $\left(k_{X}^{\mathrm{SS}}=M_{\mathrm{PU}}, \theta_{X}^{\mathrm{SS}}=P_{\mathrm{t}}\right)$ for SS, and $\left(k_{X}^{\mathrm{UPA}}=M_{\mathrm{PU}} \hat{d}, \theta_{X}^{\mathrm{UPA}}=\frac{P_{\mathrm{t}}}{\hat{d}}\right)$ for UPA. In both cases, only the scale parameter $\theta_{X}^{(\cdot)}$ depends on $P_{\mathrm{t}}$ so after finding $x_{0}$ that satisfies (8), the maximum transmit power can be solved as $P_{\mathrm{t}}=\frac{\gamma}{x_{0}}$ and $P_{\mathrm{t}}=\frac{\gamma \hat{d}}{x_{0}}$ for SS and UPA, respectively.

\section{Simulation results}

We have numerically evaluated the interference power distribution and the maximum achievable transmit powers for $M_{\mathrm{SU}}=5$ and $M_{\mathrm{PU}}=3$ antenna 
configuration. In this case, $d=5-3=2$ and for the erroneous stream number we have used $\hat{d}=3$. The maximum transmit powers were solved from (8) by using numerical integration based search with a stopping criterion of $10^{-6}$, i.e., the interference power satisfies $\left|\operatorname{Prob}\left(I_{P} \leq \gamma\right)-\left(1-P_{\text {out }}\right)\right| \leq 10^{-6}$.

First, interference power distribution was evaluated over 10000 channel realizations with $\gamma=0.3$ and $P_{\text {out }}=0.01$ and the result can be found in Fig. 1. In addition, the figure shows the corresponding interference power distribution approximations obtained in Section 3. It can be seen that the moment matching method provides a close approximation for the interference power even when only the two first moments are used. Figure 2 shows the interference outage probabilities for SS and UPA and the result confirms that both methods can satisfy the outage power constraint.

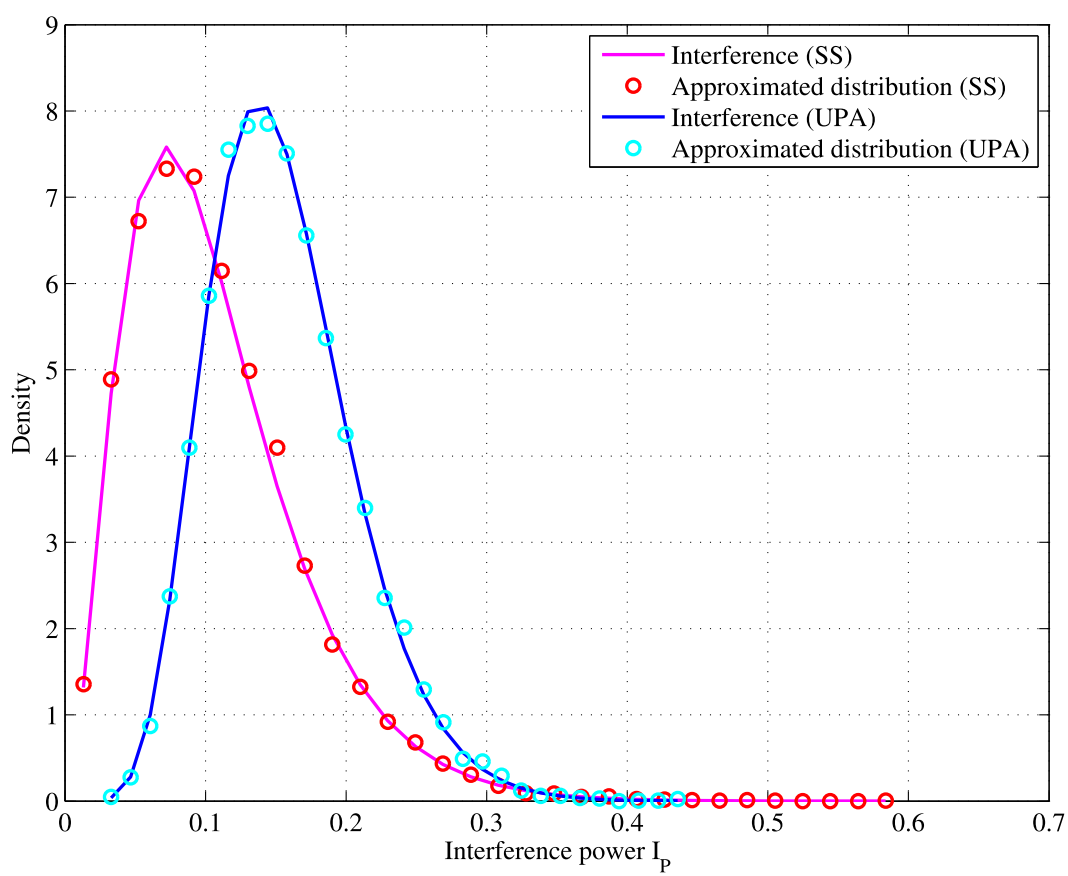

Fig. 1. Empirical interference power distribution and the corresponding approximations with $\gamma=0.3$ and $P_{\text {out }}=0.01$.

The results for the maximum transmit power can be seen in Fig. 3 where the maximum $P_{\mathrm{t}}$ are shown as a function of the target outage probability for different values of $\gamma$. It can be seen that for small outage probabilities, UPA allows higher transmit power due to having less skewed interference distribution, however, with small transmit powers SS tends to achieve higher capacity.

While the results are somewhat discouraging as the maximum transmit powers are fairly small for reasonable values of $P_{\text {out }}$, some additional considerations should be made for more practical transmit power control. First, the probability of erroneous stream allocation was not considered here. The transmit power constraint can be further relaxed to achieve the desired interference outage probability if the probability of error is less than one. In prac- 


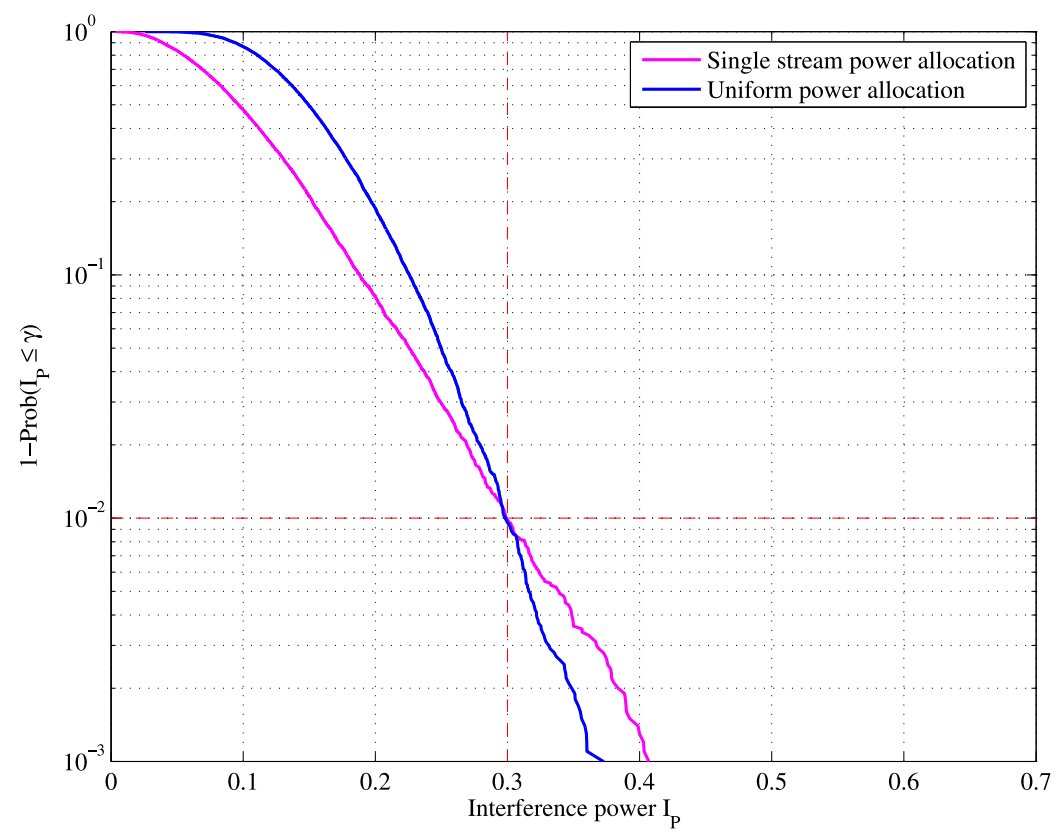

Fig. 2. Empirical interference outage probabilities with $\gamma=0.3$ and $P_{\text {out }}=0.01$.

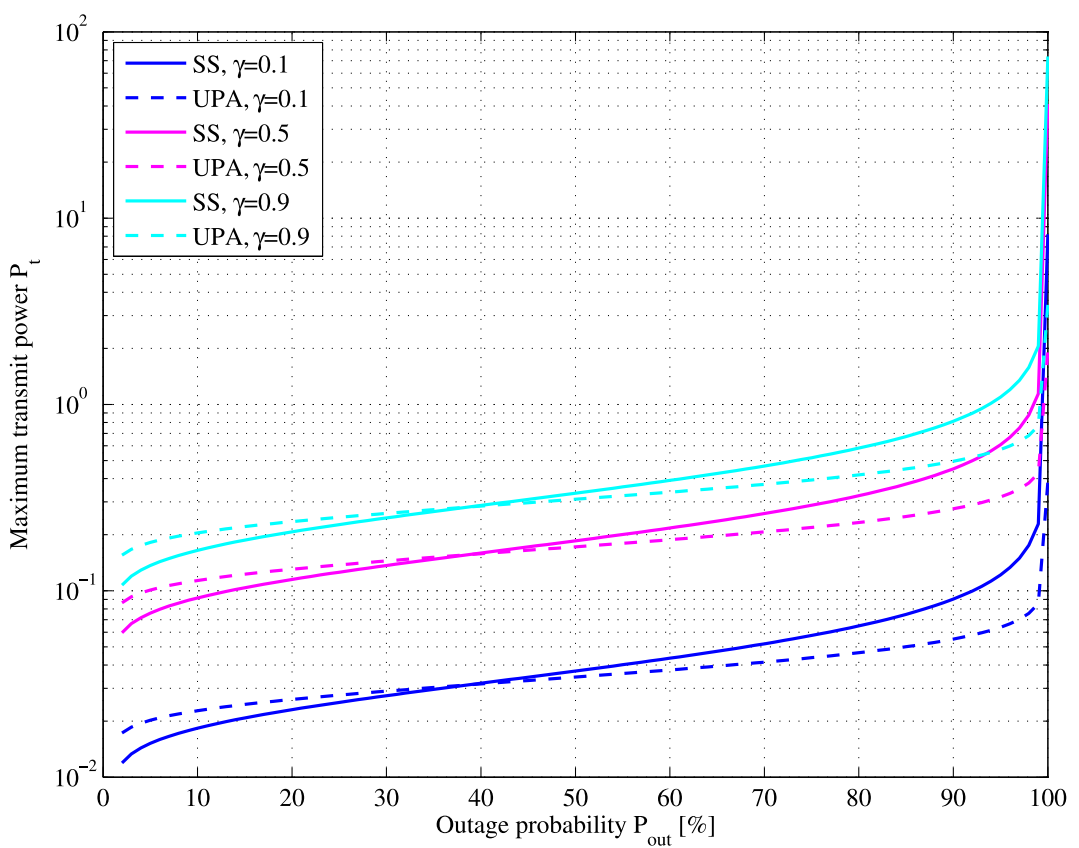

Fig. 3. Maximum achievable transmit powers as a function of outage probability for $M_{\mathrm{SU}}=5, M_{\mathrm{PU}}=3$ and $\hat{d}=3$.

tice, high estimation probability for the number of streams can be achieved even at quite low signal-to-noise ratio (SNR) region [4]. Also, the low SNR scenario usually occurs when the PU is located far away from the SU transmitter. Therefore, it is possible to allow more transmit power for the SU if path loss attenuation is taken into account. The contribution of this paper was to provide a framework for the interference power analysis and preliminary results in terms of the achievable transmit power. Future work will 
include more realistic interference model as well as the stream allocation error probabilities.

\section{Conclusion}

In this paper, we have investigated the issue where a multiantenna enabled SU of a CR network incorrectly allocates more spatial streams for the secondary transmission than are actually available. We have analyzed the interference power caused to the PU resulting from such scenario and obtained an approximation for the interference power distribution. This distribution was used to obtain the maximum transmit power for the SU which can satisfy the PU protection constraint with desired interference outage probability. Our results show that while it is possible to protect the PU from the stream allocation error, it will cause severe degradation for the SU transmission capacity. Therefore, we can conclude that accurate estimation of the available spatial transmit resources is essential in MIMO-based spectrum sharing in order to achieve proper performance.

\section{Acknowledgments}

This research was supported by the Strategic Information and Communications R\&D Promotion Programme (SCOPE). 